\title{
The versatility of the inter-costal artery perforator (ICAP) flaps
}

\author{
Moustapha Hamdi*, Koenraad Van Landuyt, Bob de Frene, \\ Nathalie Roche, Phillip Blondeel, Stan Monstrey
}

\author{
Plastic Surgery Department, Gent University Hospital, De Pintelaan 185, 9000 Gent, Belgium
}

Received 15 April 2005; accepted 1 January 2006

\section{KEYWORDS \\ Intercostal vessels; Perforator flaps; \\ Pedicled flaps; \\ Clinical experience}

\begin{abstract}
Summary Introduction: Anatomy and classification of intercostal perforator flaps in addition to our experience with will be demonstrated for different indications. Material and methods: The intercostal vessels form an arcade between the aorta and the internal mammary vessels. Different pedicled perforator flaps can be raised on this neurovascular bundle to cover defects on the trunk. They are classified as following: dorsal intercostal artery perforator flap (DICAP); lateral intercostal artery perforator (LICAP); and anterior intercostal artery perforator (AICAP) flap.

Results: Between 2001 and 2004, 20 pedicled (ICAP) flaps were harvested in 16 patients. The indications were: immediate partial breast reconstruction in eight patients who had a quadrantectomy for breast cancer; midline back and sternal defects in three patients who had radical excisions for a dermatofibrosarcoma or malignant melanoma; and autologous breast augmentation (four bilateral and one unilateral flap) in five post-bariatric-surgery patients. The average flap dimension was $18 \times 8 \mathrm{~cm}^{2}$ (range $8 \times 5-24 \times 12 \mathrm{~cm}^{2}$ ). There were two DICAP flaps, two (AICAP) flaps and 16 (LICAP) flaps. All but two flaps were based on one perforator. Mean harvesting time was 45 min for a single flap. Bilateral breast augmentation with LICAP flap necessitated longer operative time (range 2-3 h) depending whether it was combined or not with mastopoexy. Complete flaps survival was obtained. All donor sites were closed primarily.

Conclusion: The (ICAP) flaps provide valuable options in breast surgery; and for challenging defects on the trunk without sacrifice of the underlying muscle.

(c) 2006 The British Association of Plastic Surgeons. Published by Elsevier Ltd. All rights reserved.
\end{abstract}

The use of the intercostal neurovascular pedicle to supply a skin-muscle flap has been reported since a

\footnotetext{
* Corresponding author. Tel.: +329240 60 40; fax: +329240 3899.

E-mail address: moustapha.hamdi@ugent.be (M. Hamdi).
}

long-time. ${ }^{1-4}$ However, the clinical application of the intercostal vessels has not been understood before the extensive anatomical studies of Kerrigan and Daniel. ${ }^{2,4,5}$ These authors described intercostal myocutaneous flaps, with random extensions beyond the thoracic cage. Badran was the first to describe the lateral intercostal fasciocutaneous 


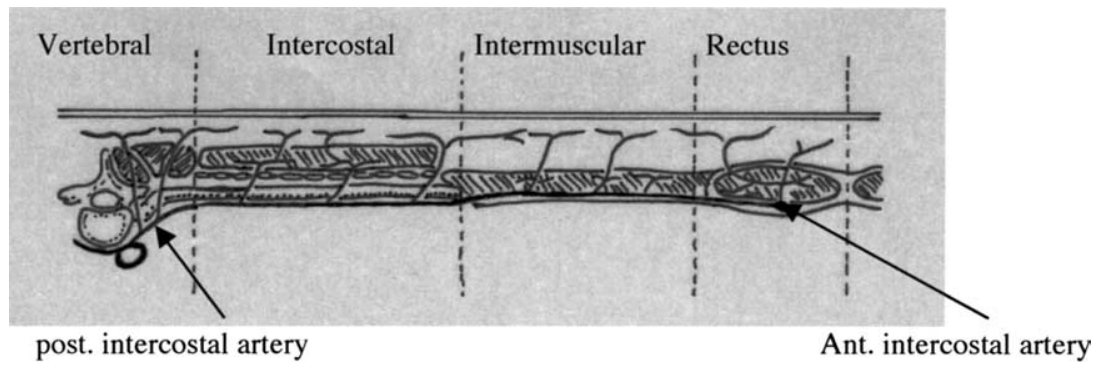

Figure 1 The course of the intercostal vessels can be divided into four segments: vertebral, intercostal, intermuscular and rectal.

free flap based on one neurovascular bundle sparing the abdominal musculature, this being, the first perforator-based intercostal free flap. ${ }^{6}$ However, pedicled intercostal perforator flaps have not been widely reported in the literature. All reported cases of intercostal flaps, either fasciocutaneous or musculocutaneous, were harvested from the lower trunk as pedicled flaps for lumbosacral defects or as free flaps for distant regions. ${ }^{1-8}$ An overview of the history, anatomy, definition and surgical technique of the intercostal artery perforator flaps are presented. The versatile clinical use of the intercostal artery perforator flaps for defects over the high trunk is demonstrated.

\section{Anatomy}

\section{Posterior intercostal bundle}

The anatomy and course of the intercostal vessels have been thoroughly studied in the anatomical work of Kerrigan and Daniel. ${ }^{5}$ There are usually nine pairs of posterior intercostal arteries in the lower nine intercostal spaces which originate from the aorta. The first and second spaces are supplied by the superior intercostal artery arising from the costocervical trunk. Every artery is accompanied by a vein of similar diameter lying above it, while the nerve lies below it.

The course of the intercostal vessels can be divided into four segments (Fig. 1). The vertebral segment is $8 \mathrm{~cm}$ long and has three branches: the dorsal branch, a nutrient branch to the rib and the collateral branch that parallels the main vessel more caudally.

In the intercostal segment $(12 \mathrm{~cm})$ the intercostal neurovascular bundle lies deep to the external and internal intercostal muscles and superficial to the innermost intercostal muscle and parietal pleura. This segment is very important because the vessels give rise to between 5 and 7 musculocutaneous perforators at intervals of
$1-3 \mathrm{~cm}$. The major musculocutaneous perforators are approximately $0.8 \mathrm{~mm}$ in diameter. At the level of the midaxillary line, the vessels and nerve give off their lateral cutaneous branches. The anatomy of these lateral cutaneous branches was widely studied on cadavers by Badran et al. ${ }^{6}$

The third segment is the intermuscular segment $(12 \mathrm{~cm})$, beginning from the costal attachment of the abdominal muscles towards the lateral border of the rectus muscle. The bundle travels between the internal oblique and transverse abdominis muscle.

The last segment is the rectus segment. The neurovascular bundle lies deep to the rectus muscle and at the midpoint of the muscle, it anastomoses with the deep epigastric vessels.

\section{The anterior intercostal bundles}

The anterior intercostal artery originates from the internal mammary artery (1st-6th space) and from its musculo-phrenic branch (7th-9th space). The anterior intercostal artery is absent in the lowest two spaces. Perforators pierce the upper 5 or 6 intercostal spaces. The anterior perforators supply the skin and the pectoralis major muscle and are often directly derived from the internal mammary artery.

\section{Classification of the intercostal flaps}

Perforator flaps were classified according to the nutrient artery as recommended by the 'Gent Consensus'(2002). ${ }^{9}$

The anterior intercostal arteries communicate with the posterior intercostal vessels at the anteromiddle third of the ribs. From this vascular arcade, multiple perforators originate from the posterior bundle. Therefore, we consider intercostal artery perforator flaps only these, which are based on the posterior bundle. The 'posterior' was dropped in 


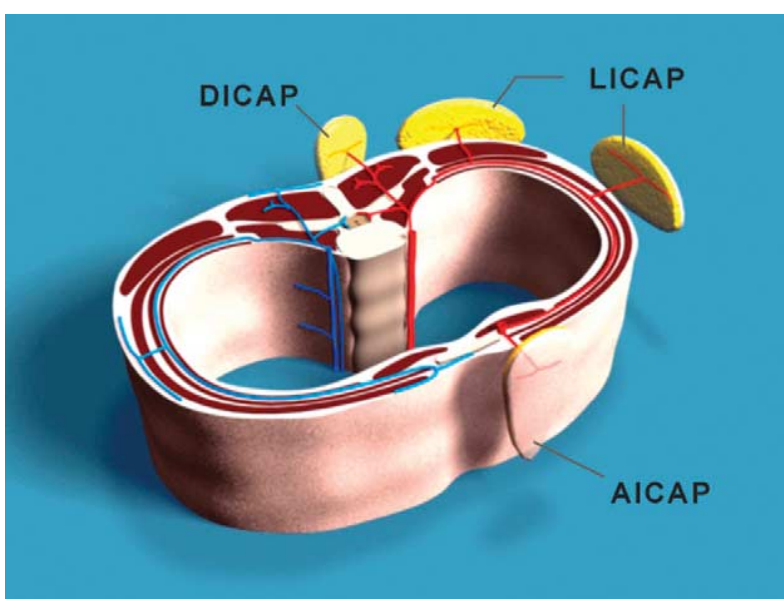

Figure 2 Many perforators originate from the intercostal vascular arcade between the aorta and the internal mammary vessels on which various perforator flaps can be raised: dorsal intercostal artery perforator (DICAP) flap, lateral intercostal artery perforator (LICAP) flap, and anterior intercostal artery perforator (AICAP) flap.

order to make the terminology of the flaps easier (Fig. 2).

Dorsal intercostal artery perforator flap (DICAP) when the flap is based on perforators arising from the vertebral segment of the intercostal vessels.

Lateral intercostal artery perforator (LICAP)

when the flap is based on perforators originating from the costal segment.

Anterior intercostal artery perforator (AICAP) flap when the flap is based on perforators that originate from the muscular or rectal segment.

\section{Operative technique}

The flap can be designed on any segment of the posterior intercostal artery. The intercostal flaps are harvested as pedicled flaps to reconstruct a distant defect of the thorax, sacrum and axillary region or as a free flap, but dissection of the vessels should then be extended into the costal groove in order to obtain a longer pedicle with a larger diameter.

\section{Dorsal intercostal artery perforator(DICAP) flap}

This flap is based on the dorsal branch of the posterior intercostal artery. It can be dissected as a free flap because the branch has an artery of $1.5 \mathrm{~mm}$ in diameter. A recent anatomical study by Ogawa et al. ${ }^{10}$ showed that the dominant dorsal intercostal perforator was the seventh intercostal perforator followed by the sixth in 57 and $30 \%$ of cases, respectively. Therefore, it is recommended to design the flap based on these perforators when a free flap is planned. The perforator is located using a unidirectional Doppler and the flap is outlined parallel to skin lines and extended laterally. The flap can be islanded and transferred as a pedicled flap, which has a short pedicle $(2-5 \mathrm{~cm})$ and, therefore, it cannot be transferred to cover a distant defect over the trunk. However, it is still useful for midline defects on the back.

\section{Lateral intercostal artery perforator (LICAP) flap}

Injection studies indicate that flaps as large as $25 \times$ $20 \mathrm{~cm}$ can be raised safely but the maximum width of donor site that can be closed primarily is $12 \mathrm{~cm} .{ }^{5,6}$ Flaps that cross the abdominal midline are dependent on a random pattern of vascular connections and should not exceed the contralateral rectus segment. The posterior border of the flap should lie at least $5 \mathrm{~cm}$ behind the posterior axillary line to make sure that the lateral cutaneous branch of the posterior intercostal arteries is included in the flap. However, it is better to locate the perforator preoperatively by using the unidirectional Doppler.

A posterior incision is made first with an anterior extension at the lower end of the flap to explore the perforators and to allow easy elevation of the flap. The incision is deepened to expose the latissimus dorsi muscle. After visualisation of the anterior border of the latissimus dorsi muscle, the smaller posterior branch of the lateral cutaneous branch is identified. This branch is followed to find the bigger anterior branch. The dissection proceeds by elevating the slip of origin of the external oblique muscle and retracting the latissimus dorsi muscle belly. Pedicle's length of $3-5 \mathrm{~cm}$ is adequate to reach a defect over the lateral or superior part of the breast, abdomen or back defects lateral to the midline. The flap can easily be folded on itself to fill a breast defect for partial breast reconstruction after a quadrantectomy.

If a longer pedicle is required for more distant defect, dissection of the main pedicle should carry on within the costal groove. The latissimus dorsi and serratus anterior muscles should be retracted to expose the interspace. Splitting these muscles in the direction of their muscular fibers may prevent the need to cut and thereby reduce blood loss and donor site morbidity. The intercostal muscles are then freed from the lower border of the rib. The 

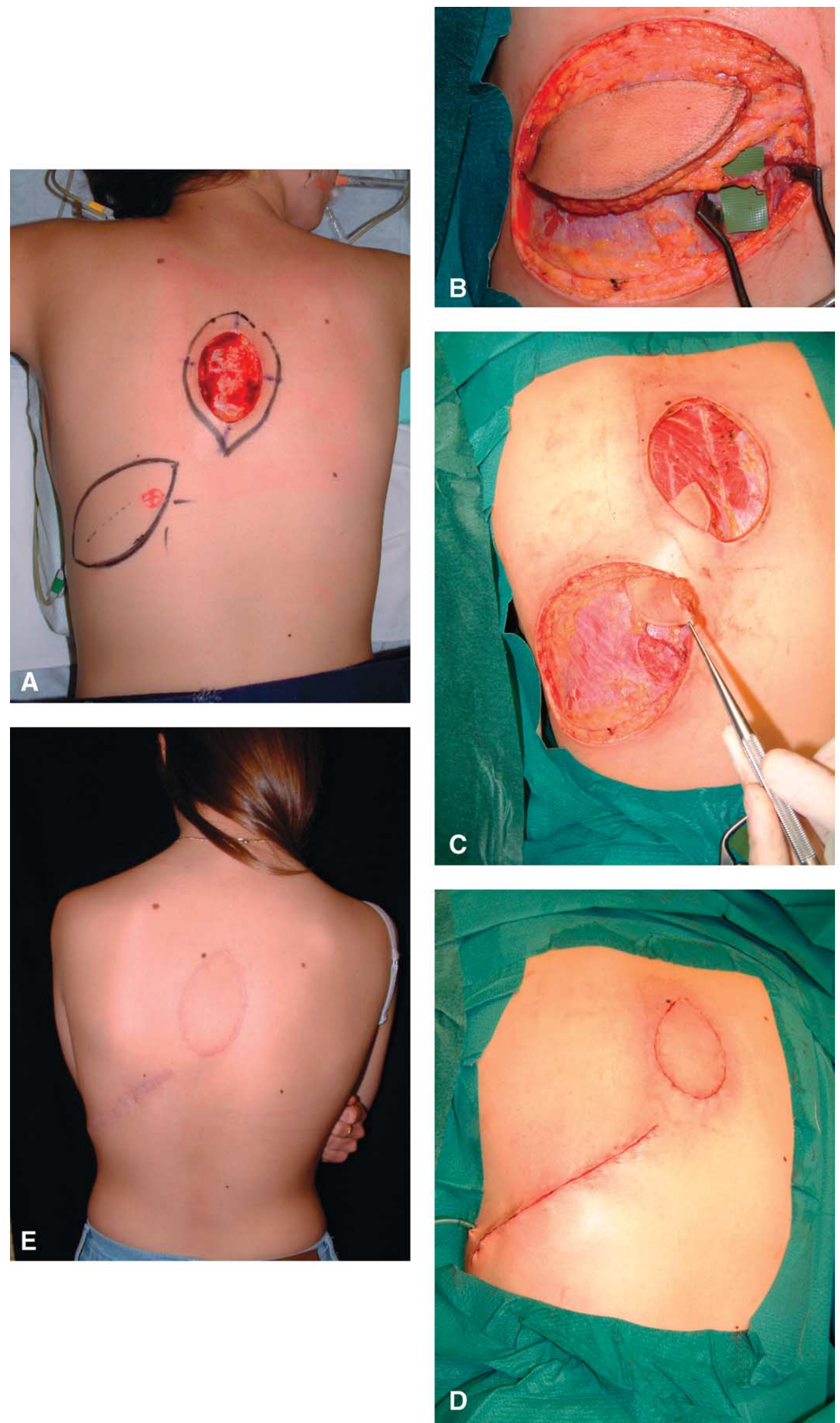

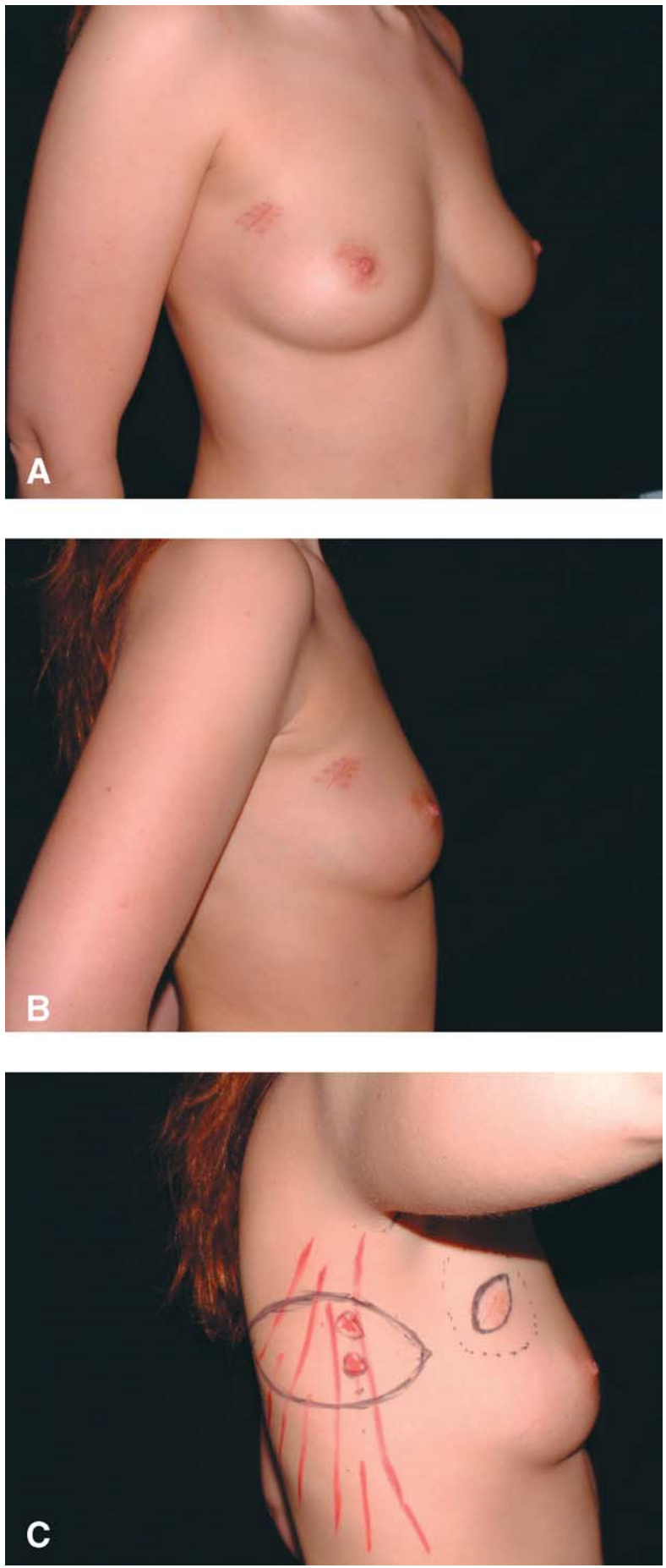

Figure 4 Partial breast reconstruction with (LICAP) flap (case 2). (A,B) Preoperative views. (C) A (LICAP) flap was designed.

periosteum is incised and reflected downwards to protect the neurovascular bundle. Care must be taken not to enter the pleural cavity. The anterior
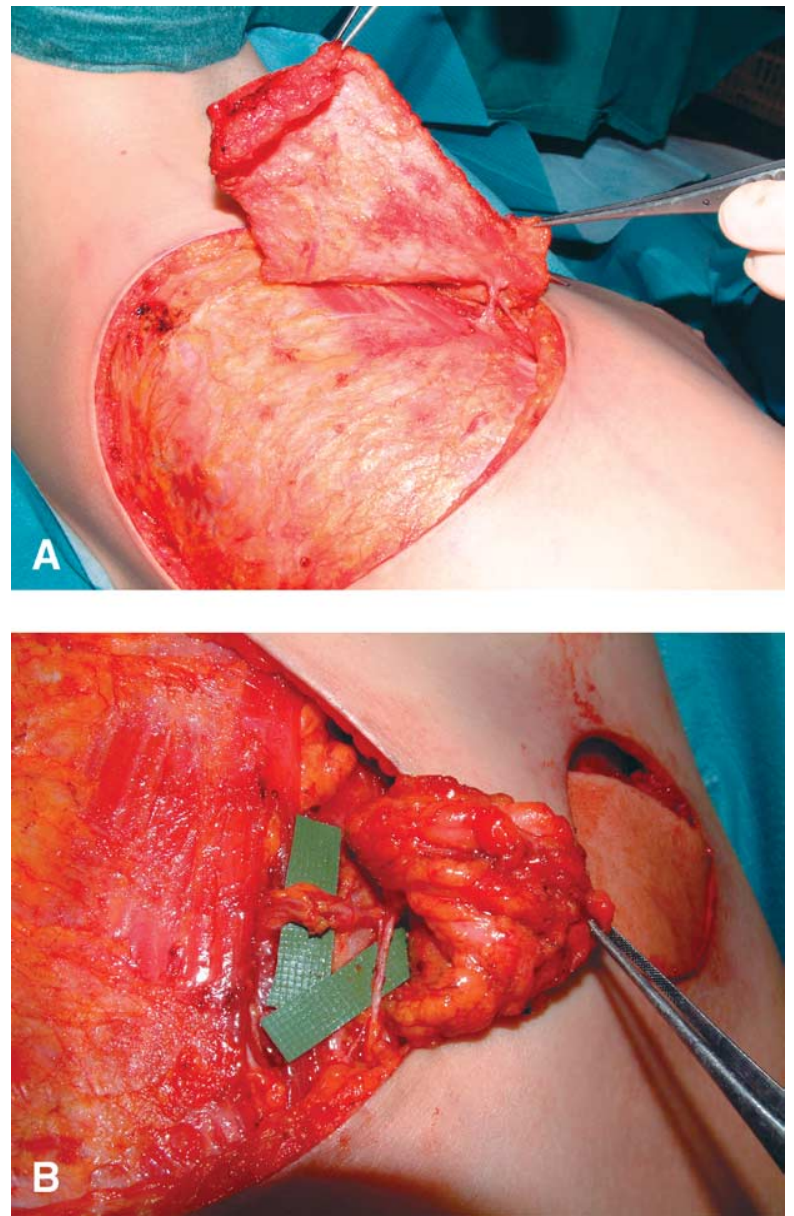

Figure 5 (LICAP) flap. (A) The flap was completely isolated on one perforator and an intercostal nerve. (B) The flap was then rotated $180^{\circ}$ and transferred into the defect.

course of the vessels is clipped after giving off the lateral cutaneous branch. The junction of the lateral cutaneous branch with the main bundle is found. The lateral cutaneous nerve can be stripped from the main intercostal nerve for any desired distance, in order to harvest a sensate flap. The rest of the flap is elevated easily above the muscle fascia.

\section{Anterior intercostal perforator(AICAP) flap}

The flap is based on a perforator of the anterior intercostal artery. The perforator can be located using a unidirectional Doppler within a $1-3 \mathrm{~cm}$ lateral to the sternal border. The flap is then designed around the perforator, usually longitudinally or obliquely towards the shoulder. The

Figure 3 A 15-year-old patient with defect on the midline (case 1): (A) the design of the DICAP flap. The Dopplered perforator was marked in red. (B) The flap was raised on two perforators. (C) The flap was passed under the skin to the defect. (D) Defect coverage and primary donor site closure. (E) Result 18 months postoperatively. 

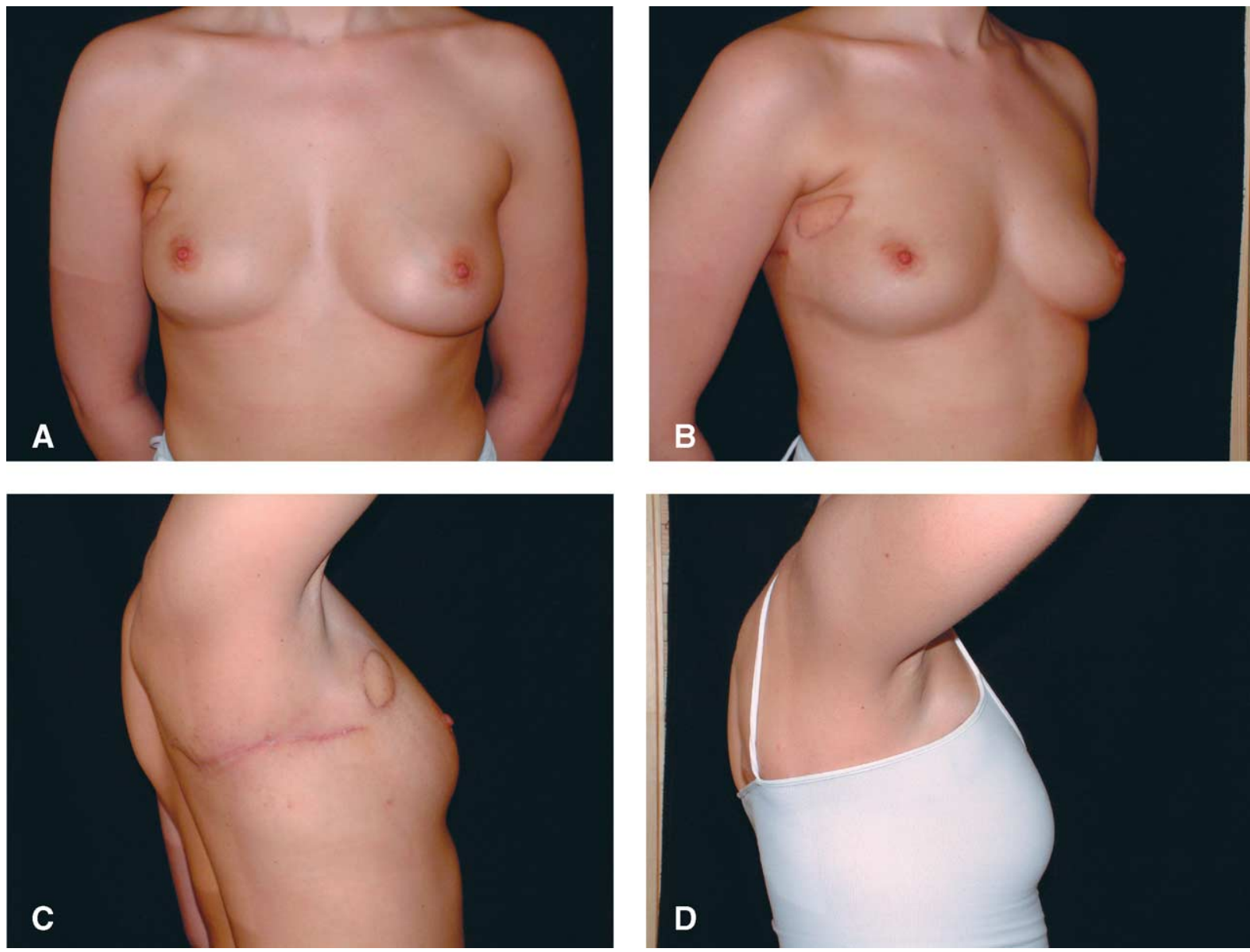

Figure 6 The results (A-D): the scar can be easily hidden under the bra.

flap is dissected from lateral to medial and from distal to cranial. The pectoralis major muscle or the rectus abdominis muscle, depending on the location of the perforator, is split and the perforator is dissected towards the anterior intercostal or internal mammary vessels. Those arising from the internal mammary vessels are found closer to the sternal margin and are larger in size especially at the level of the second and third intercostal spaces. The flap can be used for sternal, breast or thoracic defects as a transposition island flap. It can also be transferred as $\mathrm{V}-\mathrm{Y}$ advancement flap for distal or lateral defects.

\section{Material and methods}

Between June 2001 and October 2004, 20 intercostal artery perforator (ICAP) pedicled flaps were performed in 16 patients for different indications: partial breast reconstruction (eight patients), post Mohs surgery for dermatofibrosarcoma or melanoma on the trunk (three patients), and breast augmentation (one patient for breast asymmetry and four post-bariatric surgery).

\section{Results}

All ICA flaps were transferred successfully. Mean flap dimension was $18 \times 8$ (range $8 \times 5-24 \times 12 \mathrm{~cm}$ ). There were two DICAP flaps, two AICAP flaps and 16 LICAP flaps.

All but two flaps were based on one perforator. Mean harvesting time was 45 min for a single flap. Bilateral breast augmentation with LICAP flap necessitated longer operative time (range 2-3 h) depending whether it was combined with or without mastopexy. Dissection of perforators was done until their exit from the main pedicle. No dissection was necessary within the costal groove.

All donor sites were closed primarily. However, slight wound dehiscence, which healed spontaneously, occurred in two cases of post-bariatric surgery patients. 

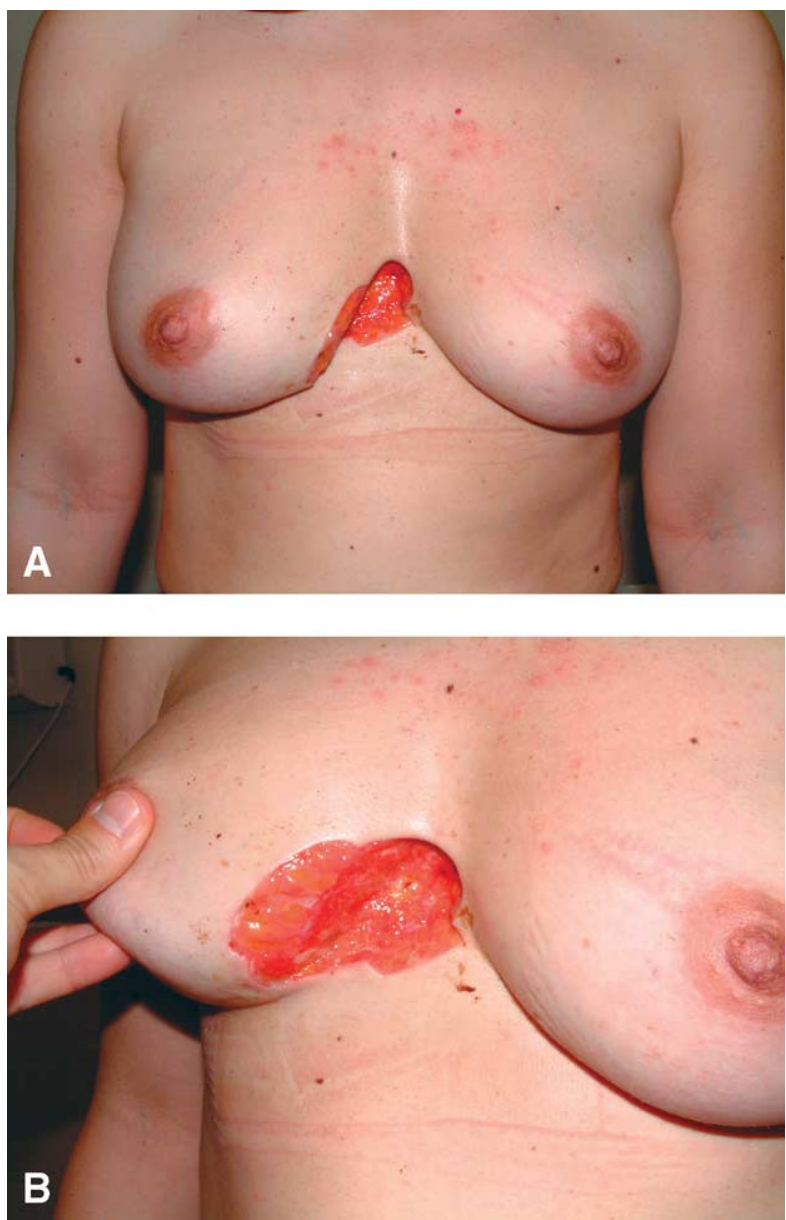

Figure 7 A 32-year-old patient with a defect after Mohs surgery (case 3$)$ : $(A, B)$ preoperative views.

\section{Case 1: (Fig. 3 (A-E))}

A 15-year-old girl who was referred to our department for wide resection after failed Mohs surgery for a dermatofibrosarcoma. The defect measured $8 \times 7 \mathrm{~cm}^{2}$ over the midline of her back. After location a perforator at the level of 7-8th intercostal spaces, a $9 \times 6 \mathrm{~cm}^{2}$ flap was drawn parallel to the rib direction (Fig. 3(A)). Two perforators were dissected through the LD muscle then through the paraspinal muscles (Fig. 3(B)). The DICAP flap was then transferred through a skin tunnel to cover the defect (Fig. 3(C,D)). Wound healing was achieved. The results were shown in Fig. 3(E).

\section{Case 2}

A 32-year-old patient was admitted for partial breast reconstruction together with further excision of breast cancer located in the supero-lateral quadrant (Fig. 4(A,B)). Two perforators were marked using a unidirectional Doppler. A $16 \times 6 \mathrm{~cm}$ flap was designed around the perforator in the bra region and parallel to the skin lines (Fig. $4(\mathrm{C})$ ). The perforator was found originated from the lateral bundle of the intercostal vessels and pierced the LD muscle at less than $1 \mathrm{~cm}$ from it anterior border. After splitting the LD muscle, one perforator was dissected within the serratus anterior muscle, intercostal muscles until its origin from the main pedicle (Fig. 5(A)). The accompanying intercostal nerve was included within the flap (Fig. $5(B)$ ). The LICAP flap was then isolated on this perforator. The flap was partially deepithelialised and folded to fill the defect. The donor site was closed primarily after undermining the skin edges. Wound healing was uneventful and the patient was referred for radiotherapy after 3 weeks. The results are shown in Fig. 6.

\section{Case 3}

A 32-year-old patient was referred to us after Mohs surgery for dermatofibrosarcoma with involved margins (Fig. 7). The resulting defect measured $8 \times 8 \mathrm{~cm}^{2}$ and extended over the presternal region and the right breast (Fig. 8(A)). An AICAP flap was designed under the right inframammary fold (Fig. 8(B,C)). The flap was islolated and $V-Y$ transferred to cover the defect. Subcutaneous stitches to the thoracic wall were placed to recreate the infra-mammary fold. The donor site was closed after undermining of the abdominal skin (Fig. 8(D)). The postoperative period was uneventful. An adequate breast contour was achieved (Fig. 9).

\section{Discussion}

The use of the intercostal neurovascular pedicle to supply a skin flap is not new in flap surgery because it has been suggested by Esser ${ }^{1}$ since 1931. In 1974, Dibbell $^{3}$ reported one clinical case of a young paraplegic patient with a sacral bed sore. The author used an intercostal flap including the anterior cutaneous nerve to provide the sensation to the sacral area. Afterward, the lateral cutaneous branch of the nerve was included into intercostal musculocutaneous flaps by Daniel et al. ${ }^{2,4}$ The flaps were harvested as muculocutaneous flaps, with random extensions beyond the thoracic cage, which needed delay procedures. The extensive anatomical studies of Kerrigan and Daniel ${ }^{5}$ resulted in a better understanding of the clinical indications and surgical technique. 

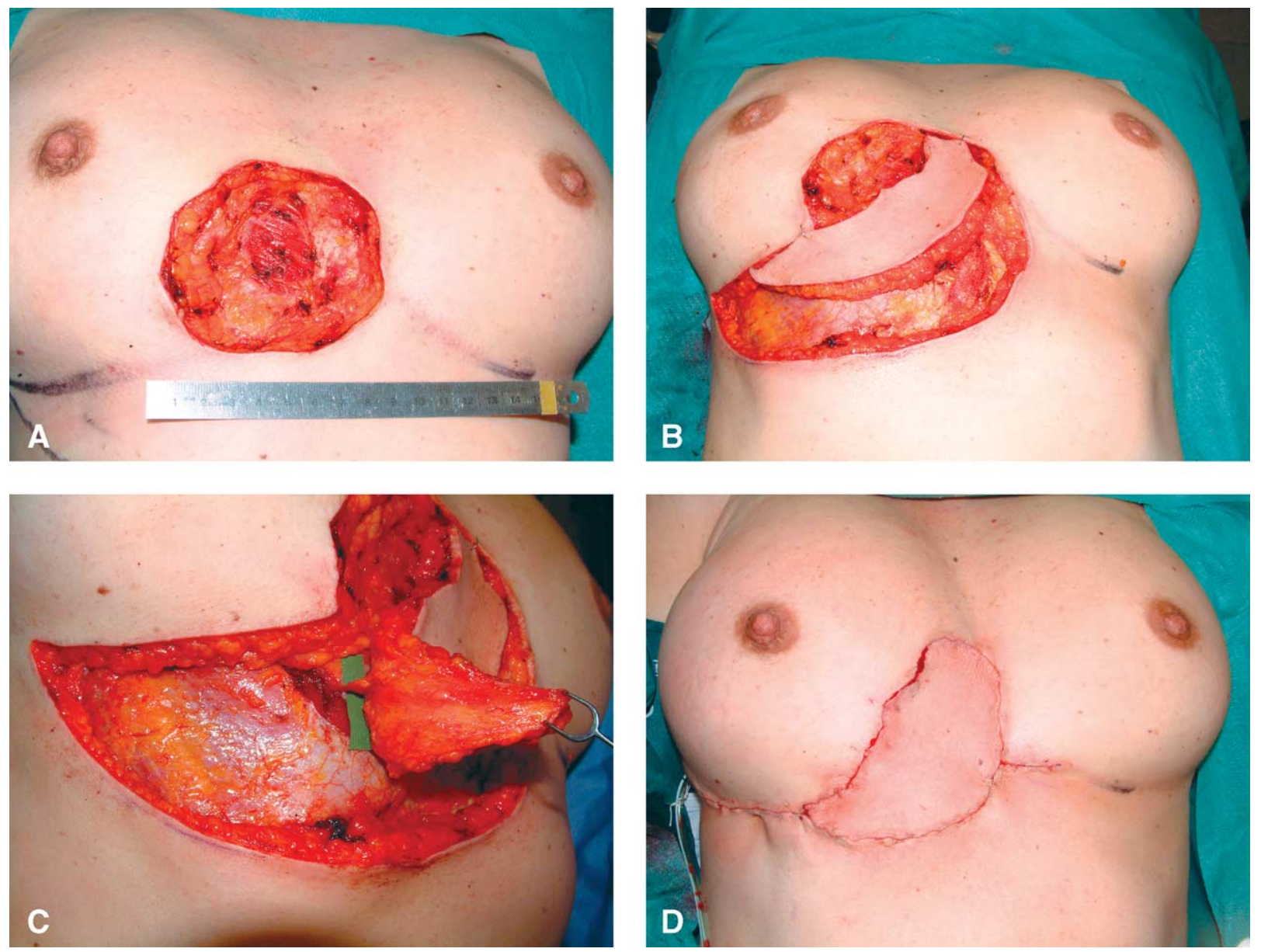

Figure 8 (A) The defect after wider excision. (B) AICAP flap was dissected under the right inframmmary fold. (C) The flap was harvested on one perforator. (D) Complete closure of the wounds.

Badran $^{6,7}$ was the first to describe the lateral intercostal fasciocutaneous flap based on one neurovascular bundle, sparing the abdominal musculature. However, intercostal flaps have not been widely reported in the literature because dissection of the main pedicle is difficult with potential incidence of pneumothorax. In addition, the dissection necessitates section of different muscles such as latissimus dorsi, external oblique and intercostal muscles.

With the introduction of the perforator concept in flap surgery, ${ }^{11,12}$ many of the previously described musculocutaneous flaps could be harvested as perforator flaps with conservation of the underlying muscle. Intercostal artery perforator (ICAP) flaps have been limited to case report publications. ${ }^{6-8,13}$ The intercostal vessels form an arcade, between the aorta and the internal mammary vessels, which gives numerous perforators. Every single perforator can be a blood supply to a skin flap. Based on the concept of free style flap, ${ }^{14}$ ICAP flaps can be harvested to cover defects that extend on the trunk from the lower neck to lower abdomen and lumbosacral area. In this series, we described the surgical technique and new indications for ICAP pedicled flaps. These flaps can be raised close to the defect, and are able to reach the defect without the need to dissect the main pedicle within the intercostal space.

The largest application of ICAP flaps is in breast surgery. ${ }^{15,16}$ We reported the first use of the LICAP in partial breast reconstruction within a clinical algorithm based on the location of the defect and the availability of these perforators. ${ }^{15}$ This flap is suitable for defects, which extend on the lateral quadrants of the breast. The perforators are located in front of the anterior border of the latissimus dorsi muscle. The dissection of the perforators is quite easy and quick; and it provides adequate perforator length to rotate the flap $180^{\circ}$ without torsion of the perforator. It is not necessary to extend the dissection into the costal groove.

Other indications for the ICAP flaps are defects over the midline of the trunk. These defects are difficult to be covered with regional traditional skin flaps. Musculocutaneous flaps used to be the first 

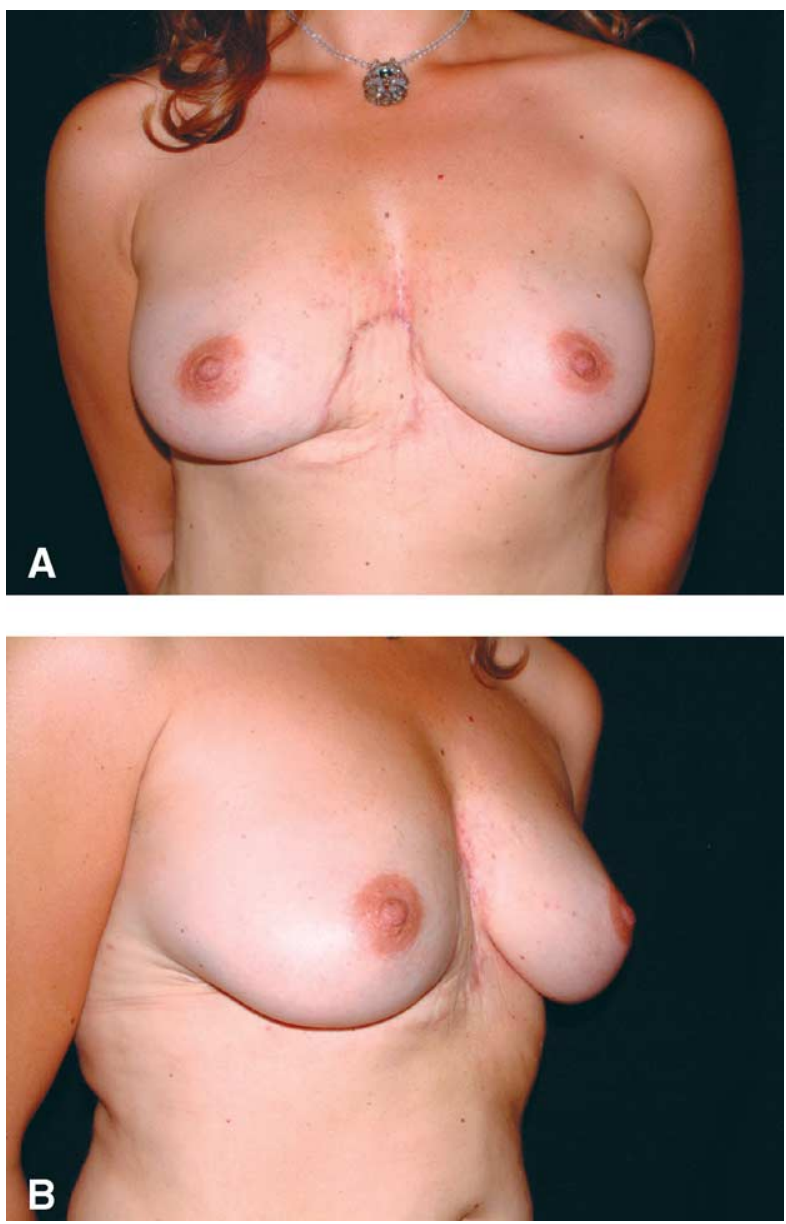

Figure 9 (A) and (B) Results at 18 months postoperatively.

choice such as latissimus dorsi to cover defects on the dorsal or lumbar region ${ }^{17}$ or the rectus abdominis for sternal defects, ${ }^{18}$ which necessitate sacrifice of large muscles. Using the ICAP flaps provides adequate cover without sacrificing any muscle and allows planning the closure of the donor sites in inconspicuous sites. Moreover, raising the ICAP flaps is quit quick and almost bloodless. Both early and long-term morbidity of the ICAP flaps are by far less than the classical musculocutaneous flaps. Finally, the ICAP flaps, like any other perforator flaps, can be defatted during surgery or be thinned by liposuction secondarily. Direct flap defatting provides immediate better aesthetic result in site where skin is thin such as the sternum and back midline.

The introduction of the perforator concept in flap surgery has resulted in a great progress in the field of reconstructive surgery during the last decade. The intercostal perforator flaps provide valuable options in breast surgery and for challenging defects on the trunk. Harvesting these flaps without sacrifice of the underlying muscle not only means reduced donor site morbidity, but also more freedom in composing and tailoring the flap.

\section{References}

1. Esser J. Biological or artery flaps of the face. Monaco: Institut Esser de Chirurgie Structive; 1931.

2. Daniel RK, Williams HB. The free transfer of skin flaps by microvascular anastomoses. An experimental study and a reappraisal. Plast Reconstr Surg 1973;52:16-31.

3. Dibbell DG. Use of a long island flap to bring sensation to the sacral area in young paraplegics. Plast Reconstr Surg 1974; 54:220-3.

4. Daniel RA. In: Strauch B, Vasconz L, Hall-Findlay E, editors. Intercostal neurovascular island skin flap. 2nd ed Grabb's encyclopedia of flaps, 2nd ed, vol. III; 1998, 1998. p. 1632 [chapter 414].

5. Kerrigan CL, Daniel RK. The intercostal flap: an anatomical and hemodynamic approach. Ann Plast Surg 1979;2:411-21.

6. Badran HA, El-Helaly MS, Safe I. The lateral intercostal neurovascular free flap. Plast Reconstr Surg 1984;73:17-26.

7. Badran HA, Youssef MK, Shaker A. Management of facial contour deformities with deepithelialized lateral intercostal free flap. Ann Plast Surg 1996;37:94-101 [discussion 101-5].

8. Roche NA, Van Landuyt $\mathrm{K}$, Blondeel Ph, Matton G, Monstrey S. The use of pedicled perforator flaps for reconstruction of lumbosacral defects. Ann Plast Surg 2000;45:7-14.

9. Blondeel PN, Van Landuyt KH, Monstrey SJ, Hamdi M, Matton GE, Allen RJ, et al. The 'Gent' consensus on perforator flap terminology: preliminary definitions. Plast Reconstr Surg 2003;112:1378-83.

10. Ogawa R, Hyakusoku H, Murakami M, Apki R, Tanuma K. An anatomical and clinical study of the dorsal intercostal cutaneous perforators, and application to free microvascular augmented subdermal vascular network (ma-SVN) flaps. Br J Plast Surg 2002;55:396-401.

11. Kroll SS, Rosenfield L. Perforator-based flaps for low posterior midline defects. Plast Reconstr Surg 1988;81: 561-6.

12. Koshima I, Moriguchi T, Soeda S, Kawata S, Ohta S, Ikeda A. The gluteal perforator-based flap for repair of sacral pressure sores. Plast Reconstr Surg 1993;91:678-83.

13. Gao JH, Hyakusoku H, Inoue S, Aoki R, Kanno K, Akimoto M, et al. Usefulness of narrow pedicled intercostal cutaneous perforator flap for coverage of the burned hand. Burns 1994; 20:65-70.

14. Wei FC, Mardini S. Free-style free flaps. Plast Reconstr Surg 2004;114:910-6.

15. Hamdi M, Van Landuyt K, Monstrey S, Blondeel P. Pedicled perforator flaps in breast reconstruction: a new concept. $\mathrm{Br}$ J Plast Surg 2004;57:531-9.

16. Van Landuyt K, Hamdi M, Blondeel P, Monstrey S. Autologous breast augmentation by pedicled perforator flaps. Ann Plast Surg 2004;53:322-7.

17. Bostwick 3rd J, Scheflan M, Nahai F, Jurkiewicz MJ. The 'reverse' latissimus dorsi muscle and musculocutaneous flap: anatomical and clinical considerations. Plast Reconstr Surg 1980;65:395-9.

18. Oh AK, Lechtman AN, Whetzel TP, Stevenson TR. The infected median sternotomy wound: management with the rectus abdominis musculocutaneous flap. Ann Plast Surg 2004;52:367-70. 